\title{
INTERFACIAL STRENGTH IN THERMOPLASTIC COMPOSITES - AT LAST AN INDUSTRY FRIENDLY MEASUREMENT METHOD ?
}

\section{J. L. Thomason, Owens Corning Automotive Solutions Centre,} Route de Charneux, B-4651 Battice, Belgium

Keywords: Glass fibre, thermoplastic, interface, fibre strength, injection moulding

\begin{abstract}
Many elegant techniques have been developed for the quantification of composite micromechanical parameters in recent years. Unfortunately most of these techniques have found little enthusiastic support in the industrial product development environment, where they are viewed as time consuming, complex, inefficient, labour intensive, and in many cases unproven or inapplicable in 'real' systems. Despite this reaction, there is a real need for a 'user-friendly' micro-mechanics to aid the composites industry to move to the next level of development. A method for deriving values for $\tau$ (the interfacial shear strength) and $\eta_{\mathrm{o}}$ (a fibre orientation factor) from a simple combination of the composite tensile stress-strain curve and the fibre length distribution has been available for some time. Despite the recent wealth of activity in the development of micro-mechanical test techniques there has been little follow-up on this older technique. In this paper we explore this analysis by its application to injection moulded glassfibre-reinforced thermoplastic composites produced using three matrices (polypropylene, polyamide 6,6 and polybutyleneterepthalate) and containing different levels of glass-fibre. We furthermore show how the analysis can be extended to obtain another important micromechanics parameter, $\sigma_{\text {uf }}$ - the fibre stress at composite failure. Values of $\tau$ and $\eta_{\mathrm{o}}$ obtained using this improved version of the original model are presented and discussed.
\end{abstract}




\section{INTRODUCTION}

There has been a rapid growth in the development and application of fibre-reinforced thermoplastic polymer composites in recent years. Parallel to this growth has been the increasing recognition of the need to better understand and measure the micro-mechanical parameters which control the strucure-property relationships in such composites. The properties of thermoplastic composites result from a combination of the fibre and matrix properties and the ability to transfer stresses across the fibre-matrix interface. Variables such as the fibre content, aspect ratio, strength, orientation and the interfacial strength are of prime importance to the final balance of properties exhibited by injection moulded thermoplastic composites. Fibre strength may be reduced significantly after fibre formation, by damage caused during both the fibre and composite production processes. Although there has not been any direct measurement of the residual strength of fibres in a moulded composite part, there is a growing body of indirect evidence that the strength of glass fibres has been significantly reduced by the time that they actually become the load bearing component of a composite. The ability to transfer stress across the fibre-matrix interphase is often reduced to a discussion of 'adhesion' which is a simple term to describe a combination of complex phenomena on which there is still significant debate as to what it means and how to measure it. Certainly, one of the generally accepted manifestations of 'adhesion' is in the mechanically measured value of interfacial shear strength (IFSS). However, many methods of determining IFSS exist and there is no overall consensus as to which method is 'best'. This situation is further complicated by the fact that sample preparation for many of these techniques is not optimised for use with thermoplastic matrices.

Despite the elegance of the many techniques which have been developed for the quantification of composite micro-mechanical parameters, these techniques have found little enthusiastic support in the industrial product development environment. It should be clear that, the more dissimilar the experimental sample must be from the composite part the greater must be the extrapolation and consequent uncertainty between the measured result and the real composite performance. Furthermore, the higher the number of measurements that must be made for 'reliable' statistics, the longer and more labour intensive the measurement. Finally, the more complex and disputed the underlying theories supporting the analysis then, together with the foregoing, the less likely the technique is liable to gain acceptance in an industrial environment. There continues to be discussion and disagreement about many of these complex areas - which is healthy and acceptable in an academic environment - but gains little support in an industrial environment where time scales and resources are ever diminishing. It is unfortunate that many of these techniques are indeed viewed as time consuming, complex, inefficient, labour intensive, and in many cases unproven or inapplicable in 'real' systems. Consequently their application in most industrial product development programmes is rare. This leads to a classic situation where, because these methods have little support in an industrial environment, they rarely get the time and development to show their usefulness. This occurs despite the fact that the underlying science of even the most apparently mundane industrial development often necessitates solutions which require a deep understanding of structure-performance and microstructural analysis. Many 'traditional' product development strategies are reaching a level on the 'S-curve' of rapidly diminishing returns and there is a real need for a 'user-friendly' micromechanics to aid composites to move to the next level of development. In addition to access to such knowledge, composite product developers also need tools which can fit their toolbox and do not need a new and expensive workshop to house them.

Most laboratories involved in the development of thermoplastic composites will routinely measure composite mechanical properties such as tensile strength, and determine residual fibre length. A series of papers by Bader and Bowyer $(1,2)$ in the early seventies presented a method for deriving values for $\tau$ (the IFSS) and $\eta_{0}$ (a fibre orientation factor) from a simple combination of the tensile stress-strain curve and the composite fibre length distribution. It is interesting to note that, despite the recent wealth of activity in the development of micro- 
mechanical test techniques (or perhaps because of it ) there has been little follow-up to these papers. In this paper we present an improved version of this method and illustrate its application to injection moulded glass-fibre-reinforced thermoplastic composites. Furthermore, we show how the analysis can be extended to obtain another important micro-mechanics parameter, $\sigma_{\text {uf }}$ the average fibre stress at composite failure.

\section{ANALYSIS METHOD}

The analysis method of Bowyer and Bader is based on the Kelly-Tyson model for the prediction of the strength $\left(\sigma_{u c}\right)$ of a polymer composite reinforced with discrete aligned fibres (3). This model can be simplified to $\sigma_{\mathrm{uc}}=\eta_{\mathrm{o}}(\mathrm{X}+\mathrm{Y})+\mathrm{Z}$, where $\mathrm{Z}$ is the matrix contribution, $\mathrm{X}$ is the subcritical fibre contribution, and $\mathrm{Y}$ is the super critical contribution, in reference to a critical fibre length defined by $\mathrm{L}_{c}=\sigma_{\mathrm{f}} \mathrm{D} / 2 \tau$. The Kelly-Tyson model assumes that all the fibres are aligned in the loading direction and the equation cannot be integrated to give a simple numerical orientation factor to account for the average fibre orientation. The common approach to this problem is to fit the experimental data using a simple numerical orientation factor $\left(\eta_{\mathrm{o}}\right)$. Bowyer and Bader extended the original Kelly-Tyson concept to model the stress-strain curve of the composite prior to failure. The basis of their argument was that at any strain value $\left(\varepsilon_{\mathrm{c}}\right)$ there exists a critical fibre length $L_{\varepsilon}=\sigma_{f} . D / 2 \tau$. Fibres shorter than $L_{\varepsilon}$ carry an average stress $=L . \tau / 2 D$ and fibre longer than $\mathrm{L}_{\varepsilon}$ carry an average stress $=\mathrm{E}_{\mathrm{f}} \cdot \varepsilon_{\mathrm{c}}\left(1-\left(\mathrm{E}_{\mathrm{f}} \varepsilon_{\mathrm{c}} \mathrm{D} / 4 \mathrm{~L} . \tau\right)\right.$. The composite stress at any strain level was then given by

$$
\sigma_{c}=\eta_{0}\left(\sum_{i}\left[\frac{\tau L_{i} V_{i}}{D}\right]+\sum_{j}\left[E_{f} \varepsilon_{c} V_{j}\left(1-\frac{E_{f} \varepsilon_{c} D}{4 \tau L_{j}}\right)\right]\right)+\left(1-V_{f}\right) E_{m} \varepsilon_{c}
$$

Bowyer and Bader then went on to show that, although $\eta_{\mathrm{o}}$ and $\tau$ are not generally known, values for these factors can be obtained if the composite stress $\left(\sigma_{1}\right.$ and $\left.\sigma_{2}\right)$ at two strain values $\left(\varepsilon_{1}\right.$ and $\left.\varepsilon_{2}\right)$ are known.. The matrix contribution $\mathrm{Z}$ is calculated from an independent matrix modulus determination and used to calculate the ratio $\mathrm{R}$ of the fibre contributions at the two strains

$$
R=\frac{\sigma_{1}-Z_{1}}{\sigma_{2}-Z_{2}} \quad R^{\prime}=\frac{X_{1}+Y_{1}}{X_{2}+Y_{2}}
$$

Equation 1 was then used with an assumed value of $\tau$ to calculate the ratio $\mathrm{R}$ ' the theoretical value of $R$. At this point the calculations are independent of $\eta_{o}$. The value of $\tau$ is then adjusted until $R^{\prime}=R$, and that value of $\tau$ is then used in Equation 1 to obtain a value for $\eta_{o}$ (which is assumed to be the same at both strain levels).

It should be realised that all available 'micro-mechanical' methods for obtaining values such as orientation factor and interfacial interaction parameters require a long list of assumptions to be taken into account, and this method is no different in that respect $(4,5)$. However, the method presented here has an enormous attraction in that it utilises data which are readily available from the standard composite mechanical testing and requires only an extra determination of fibre length distribution, which is a common characterisation tool of those working with discontinuous fibre composites. At the time of the original work the method was somewhat time consuming due to the limited computer power available, however nowadays the above equations can be reduced to a simple spreadsheet operation where $\tau$ and $\eta_{\mathrm{o}}$ can be obtained in moments. We have also extended the analysis method to obtain a value for $\sigma_{u f}$ the fibre stress at composite 
failure. This can be obtained by inserting the composite breaking stress and strain into the original Kelly-Tyson equation along with the determined values of $\tau$ and $\eta_{\mathrm{o}}$.

Furthermore, in the original analysis it was assumed that the matrix stress contribution could be calculated from the matrix stiffness and the composite strain (as shown in equation 1). It is well known that the stress-strain curve of many thermoplastics is non-linear, even at low strains. We have found it more expedient to obtain polynomial curve fitting parameters for the stress-strain curves of our different matrix polymers between 0-3\% strain (few composites exceed this range). The matrix contribution can then easily be calculated for any strain level we wish to use in the analysis. Consequently, this method can give a complete characterisation of the micromechanical parameters $\eta_{\mathrm{o}}, \tau, \sigma_{\text {uf }}$ of any system. The relative simplicity and cost effectiveness of this approach makes it ideal as an industrial screening tool for product developers. We further illustrate the use of this method below by its application to a study of the mechanical performance and micromechanical parameters of injection moulded glass fibre reinforced thermoplastics.

\section{EXPERIMENTAL}

The E-glass samples in this study were all Owens Corning Cratec ${ }^{\mathrm{TM}} 4 \mathrm{~mm}$ chopped strands. Glass samples were chosen all nominal $14 \mu \mathrm{m}$ diameter to eliminate diameter as a variable. The polymer matrix materials studied were Polyamide 6,6 (PA66), Polybutyleneterephthalate (PBT), Polypropylene homopolymer $(\mathrm{PPh})$ and Polypropylene containing 2\% of maleated-PP coupling agent $(\mathrm{PPm})$. The glass and polymer product numbers are identified in Table 1 along with the compounding and moulding conditions. The glass bundles and pre-dried polymer pellets were dry blended and compounded on a single screw extruder (2.5 inch, 3.75:1, 24:1 L/D screw). The compounds were moulded into test bars on a 200-ton Cincinnati Milacron moulding machine. All mechanical property testing was performed at $23^{\circ} \mathrm{C}$ and at a relative humidity of $50 \%$, specimens were tested 'dry as moulded'. Tensile properties were measured in accordance with the procedures in ASTM D-638, at a crosshead rate of $5 \mathrm{~mm} / \mathrm{min}(0.2$ inches $/ \mathrm{min})$ and an extensometer gauge length of $50 \mathrm{~mm}$ ( 2 inches). Fibre length and diameters were determined by image analysis and optical microscopy on fibre samples removed from the moulded bars after high temperature ashing. Measurement of fibre orientation was carried out on cross sections of moulded tensile bars cut perpendicular to the flow direction. The sections were polished and a series of optical micrographs was taken systematically across the thickness of the bar. The orientation of any fibre $(\phi)$ with respect to the flow direction can be determined from its elliptical profile (6). The average value of $\cos ^{2}(\phi)$ can be used to represent the average of the orientation distribution of the fibres in the sample and is approximated by

$$
<\cos ^{2}(\phi)>=\sum_{\mathrm{i}}\left[\mathrm{N}\left(\phi_{\mathrm{i}}\right) \cos ^{2}\left(\phi_{\mathrm{i}}\right)\right] / \sum_{\mathrm{i}}\left[\mathrm{N}\left(\phi_{\mathrm{i}}\right)\right]
$$

However, it should be realised that although $\left\langle\cos ^{2}(\phi)\right\rangle$ may be considered as an orientation factor it is not necessarily unique or expected (a priori) to take the same value as $\eta_{\mathrm{o}}$ used in the macro-mechanical analysis presented above.

\section{RESULTS AND DISCUSSION}

The tensile properties of the three injection moulded polymers are presented in Table 2 along with the parameters from the regression analysis of the stress-strain curves between 0-3\% strain. The matrix stress at any strain value ( $\varepsilon$ in $\%$ strain) is obtained from

$$
\sigma_{\mathrm{um}}=\mathrm{X}_{3} \varepsilon^{3}+\mathrm{X}_{2} \varepsilon^{2}+\mathrm{X}_{1} \varepsilon
$$


The properties of the polymers shown in Table 2 are well known, although it is of interest to note the drop in polypropylene (PP) modulus caused by the addition of the coupling agent. The data from the characterization of the moulded composites is shown in Table 3. The performance ranking of these composites (PA66 $>\mathrm{PBT}>\mathrm{PPm}>\mathrm{PPh}$ ) is also as expected. The strength of the GF-PP moulding is significantly increased by the addition of the coupling agent. Figure 1 shows the stress-strain data at the points selected for the micro-mechanical analysis and at composite failure. It is interesting to note that the limited data is this Figure reflects the trend seen in the full stress-strain curves of the two PP based composites. The lower modulus of the polymer PPh is reflected in the lower modulus of the GF-PPh composite. Otherwise the stressstrain curves of the PP based composites are very similar up to the failure of GF-PPh. This indicates that the principle effect of the addition of PP coupling agent appears to be to increase the strain to failure of the composite. For this reason we chose our strain values for the micromechanical analysis to be at approximately $1 / 3$ and $2 / 3$ of the average strain to failure of the composites rather than at fixed values, the actual values are given in Table 3 . We also show the number and weight average fibre lengths obtained from analysis of the moulded composites in Table 3. In this set of samples there appears to be an inverse relationship between the residual fibre length and the composite strength, i.e. the GF-PA66 sample has the highest strength despite having the shortest fibres. Using the values presented in Table 3, and the full fibre length distribution from each sample, we obtain the values for $\eta_{\mathrm{o}}, \tau, \sigma_{\mathrm{uf}}$ in each system as shown in Table 4. The values for $\tau$ are ranked in the order that one might expect from the fibrematrix combinations. At this stage the meaning of the absolute values is open to discussion (as is the case for most other values of interfacial interaction parameter) although we can assume that they may be used in the Kelly-Tyson model to make predictions of composite strength for each of these systems. Notwithstanding this limitation, the method clearly has potential as a cost effective method for screening interfacial effects such as might be expected from the application of different sizing systems.

A number of authors have commented on the role of shrinkage stresses contributing to the stress transfer capability at the interface (4-10). Most composite materials are shaped at elevated temperature and then cooled. Since in most cases the thermal expansion coefficients of thermoplastic polymers are much greater than reinforcement fibres this cooling process results in compressive radial stress $\sigma_{R}$ at the interface. Assuming that the coefficient of friction $(\beta)$ at the interface is non-zero these compressive stresses will contribute a frictional component $\tau_{\mathrm{f}}=\beta . \sigma_{\mathrm{R}}$ to the apparent shear strength of the interface. In the case of thermoplastic polymer matrices where there may often be little or no chemical bonding across the interface these frictional stresses can make up a large fraction of the apparent IFSS. An exact calculation of the frictional fraction of the IFSS requires detailed knowledge of the interfacial friction and the temperature dependence of the stiffness and thermal expansion coefficient of the composite constituents and is beyond the scope of this study. However, we have estimated the magnitude of $\sigma_{R}$ in our glass reinforced thermoplastics using a number of different models and fixed room temperature values of the required input parameters (8-10). The results from all three models follow the same general trends, differing only in the absolute level of radial stress predicted. In Figure 2 we compare the results from Nairns model for radial shrinkage stress (no interphase present) with the IFSS values obtained from the macro-model. The relevant model input values are shown in Table 5. It can be seen that results follow a similar trend. By an appropriate choice of coefficient of friction it is possible to obtain a good fit of any of the three models with the experimental data. Figure 2 also shows an example of the predictions of Nairn's model combined with $\beta=0.67$ to give an estimate of the frictional component of the IFSS. It can be seen that we get excellent agreement with the experimental data. At this point we stress that we do not wish to imply that any strong relevance should be attached to the value of $\beta$ since such an analysis requires a much greater level of detail (i.e. temperature dependence of many parameters) before it can be 
considered realistic. However the excellent agreement in the observed trends does imply that shrinkage stress and interfacial friction may play an important role in the IFSS of these materials.

Figure 3 compares the orientation factors obtained from the macro-method with $\left\langle\cos ^{2}\right\rangle$ values obtained by optical analysis of polished cross sections as described by equation 3 . It is also possible to calculate an orientation factor from the individual composite modulus data using a simple rearrangement of the "rule-of-mixtures" equation

$$
\eta_{\mathrm{o}}=\left(\mathrm{E}_{\mathrm{c}}-\mathrm{V}_{\mathrm{m}} \mathrm{E}_{\mathrm{m}}\right) / \eta_{\mathrm{l}} \mathrm{V}_{\mathrm{f}} \mathrm{E}_{\mathrm{f}}
$$

where $\eta_{1}$ is the length factor in the Cox shear lag model $(11,12)$. These values are also included in Figure 3. There does not appear to be any strong correlation between the various methods with all values falling into the range of 0.6-0.7. The potential level of experimental error in obtaining an orientation factor from any of these methods is so large that further detailed interpretation of Figure 3 is hardly warranted.

It can be seen from Table 4 that $\sigma_{\text {uf }}$ the level of maximum fibre stress in these composites (990$1930 \mathrm{MPa}$ ) is low compared to the pristine tensile strength of E-glass (3500 MPa). However, these values are of the same order of magnitude as those recently reported by Thomason and Kalinka for the tensile strength (1500-2000 MPa) at short gauge lengths of E-glass fibres removed from chopped glass bundles similar to the input materials in this study (13). Fibre strength may be reduced significantly after fibre formation by damage caused during both the fibre and composite production processes (13-15). Although there is little available published data on the direct measurement of the residual strength of fibres in a moulded composite part, there is a growing body of indirect evidence indicating that the strength of glass fibres has been significantly reduced by the time that they actually become the load bearing component of a composite (13-16). Thomason et al. have recently demonstrated the wide range of fibre strength to be found in fibres used in GMT. Differences in fibre strength were attributed to different levels of processing damage and fibre sizing protection efficiency (15). They reported values for average E-glass fibre strength as low as $1100 \mathrm{MPa}$ in fibres extracted from commercial GMT's. Strength reducing flaws of many types can be introduced during processing either through fibrefibre contact or fibre contact with the processing equipment. Fibre length reduction during processing occurs through breakage of fibres at their weakest (flawed) point. It is interesting to note in the data in Tables 3 and 4 that the residual fibre lengths in these samples appears to be inversely related to the IFSS value obtained from the macro-model analysis. If the solid state value of $\tau$ is an indicator for the level of fibre-melt interaction during processing then we may be seeing evidence that the fibre-melt interaction level is an important parameter in determining the level of fibre length degradation during processing. One possible mechanism is that an increase in fibre-melt interaction may lead to a greater increase in apparent viscosity of the melt giving a higher level of shear and fibre breakage. Another possibility is that greater fibre-melt interaction may lead to a more rapid dispersion of the fibre bundles in the extruder. This would lead to higher viscosities and a greater number of individual fibres exposed to shear earlier in the processing which could easily be related to a greater level of fibre length degradation. This will require further investigation. The fibre stress values in Table 4 can be converted to fibre strain at failure through the fibre modulus ( $72 \mathrm{GPa}$ ). Fibre strain at composite failure calculated using the macro-method fibre stress values is compared with the experimental composite tensile strain at failure in Figure 4. The agreement is excellent. 


\section{CONCLUSIONS}

This paper has presented a method for deriving values for the interfacial shear strength, a fibre orientation factor, and the fibre stress at composite failure, from a simple combination of tensile stress-strain values and the residual fibre length distribution of injection moulded glass-fibrereinforced thermoplastic composites. The interpretation of the parameters obtained from this macro-mechanical method is no more or less open to discussion than those parameters obtained by single fibre micro-mechanical methods. However, this method is much less labor intensive and consequently more cost effective. Furthermore, it has the advantage that the values are obtained from analysis of 'real' composites. Data obtained from the macro-mechanical analysis of various injection moulded glass-fibre-reinforced thermoplastics indicated that residual interfacial radial compressive stresses may contribute significantly to the interface shear strength in thermoplastic matrix composites. The orientation factor obtained from the macromechanical analysis was in general agreement with such factors obtained by other methods. Significant differences in the fibre stress at composite failure were found dependent on the resin system. Values obtained by the macro-method were in excellent agreement with the experimental values for the composite elongation at failure. The low level of maximum fibre stress obtained was in line with other published values for this type of composite. Given the wealth of microstructural information obtained from this macroscopic analysis and the low level of resources employed to obtain the data we believe that this method deserves further investigation as a screening tool in composite system development programmes.

\section{TABLE OF SYMBOLS}

$\begin{array}{ll}\varepsilon_{\mathrm{c}} & \text { composite strain } \\ \eta_{\mathrm{o}} & \text { fibre orientation factor } \\ \sigma_{\mathrm{c}} & \text { composite stress at a given strain } \\ \sigma_{\mathrm{uc}} & \text { composite ultimate stress } \\ \sigma_{\mathrm{f}} & \text { the fibre strength } \\ \sigma_{\mathrm{uf}} & \text { the fibre stress at composite failure } \\ \sigma_{\mathrm{um}} & \text { the matrix stress at the composite failure strain } \\ \tau & \text { the interfacial shear strength } \\ \mathrm{D} & \text { average fibre diameter } \\ \mathrm{E}_{\mathrm{f}, \mathrm{m}} & \text { fibre, matrix modulus } \\ \mathrm{L}_{\mathrm{c}} & \text { critical fibre length } \\ \mathrm{L}_{\mathrm{i}, \mathrm{j}} & \text { fibre length } \\ \mathrm{V}_{\mathrm{f}} & \text { the volume fraction of fibres of length }\end{array}$

\section{REFERENCES}

1. Bowyer W.H. and Bader M.G., ' On the reinforcement of thermoplastics by perfectly aligned discontinuous fibres', J. Mater. Sci., 1972, 7, 1315-1321.

2. Bader M.G. and Bowyer W.H., 'An improved method of production for high strength fibrereinforced thermoplastics', Composites, 1973, 4, 150-156.

3. Kelly, A. and Tyson, W.R., 'Tensile properties of fibre-reinforced metals', J.Mech.Phys.Solids, 1965, 13, 329-350. 
4. Thomason, J.L., ' Micromechanical Parameters from Macromechanical Measurements on Glass Reinforced Polyamide 6,6', Composites Science and Technology 61 (2001) pp 20072016

5. Thomason, J.L., ' Micromechanical Parameters from Macromechanical Measurements on Glass Reinforced Polybutyleneterepthalate', Composites Part A 33 (2002) pp 331-339.

6. Thomason, J.L, ' The influence of fibre properties of the performance of glass-fibrereinforced polyamide 6,6.', Composites Science and Technology 59 (1999) pp 2315-2328

7. Di Landro, L. and Pegoraro, M. Evaluation of residual stresses and adhesion in polymer composites. Composites Part A, 1996, 27A, 847-853.

8. Piggott, M.R. in "Load-bearing fibre composites", Pergamon Press, Oxford 1980 pp 203209.

9. Nairn, J.A. Thermoelastic analysis of residual stresses in unidirectional, high-performance composites. Polym. Compos., 1985, 6, 123-130.

10. Cox, H.L. The elasticity and strength of paper and other fibrous materials. Brit.J.Appl.Phys., 1952, 3, 72-79.

11. Thomason, J.L. and Vlug, M.A. The influence of fibre length and concentration on the properties of glass fibre reinforced polypropylene: 1) Tensile and Flexural Modulus. Composites, 1996, 27A, 477-484.

12. Thomason, J.L. and Vlug, M.A. The influence of fibre length and concentration on the properties of glass fibre reinforced polypropylene: 4) Impact properties. Composites, 1997, 28A, 277-288.

13. Thomason J.L., Kalinka G., 'A technique for the measurement of reinforcement fibre tensile strength at sub-millimetre gauge lengths', Composites Part A 32 (2001) pp 85-90

14. Thomason, J.L. The influence of fibre properties on the properties of glass-fibre-reinforced polyamide 6,6. J. Compos. Mater., 1999, 34, 158-172.

15. Thomason, J.L., Vlug, M.A., Schipper, G. and Krikor, H.G.L.T. The influence of fibre length and concentration on the properties of glass fibre reinforced polypropylene: 3 ) Strength and strain at failure. Composites, 1996, 27A, 1075-1084.

16. Moriwaki, T. Mechanical property enhancement of glass fibre reinforced polyamide composite made by direct injection. Composites, 1996, 27A, 379-384.

Table 1 Raw materials and processing conditions

\begin{tabular}{|lcccc|}
\hline Resin & PA6,6 & PBT & PPm & PPh \\
\hline Glass & 123D-14C & 183F-14C & 146B-14C & 146B-14C \\
Matrix Polymer & DuPont & Celanese 2002- & 2\% Polybond & Huntsman \\
Compounding Extruder Set & $288-293$ & $271-299$ & $254-277$ & $254-277$ \\
$\begin{array}{l}\text { Temperatures }\left({ }^{\circ} \mathrm{C}\right) \\
\text { Injection Moulding }\end{array}$ & $293-299$ & $271-293$ & $215-238$ & $215-238$ \\
$\begin{array}{l}\text { Set Temperatures }\left({ }^{\circ} \mathrm{C}\right) \\
\text { Mould Temperature }\left({ }^{\circ} \mathrm{C}\right)\end{array}$ & 93 & 82 & 65 & 65 \\
\hline
\end{tabular}


Table 2 Resin tensile properties and stress-strain curve fitting factors

\begin{tabular}{|lcccc|}
\hline Sample & PA6,6 & PBT & PPm & PPh \\
\hline Strength $(\mathrm{MPa})$ & 70.4 & 54.3 & 32.4 & 31.9 \\
Modulus $(\mathrm{GPa})$ & 2.9 & 2.8 & 1.5 & 1.9 \\
Elongation $(\%)$ & 3.9 & 10.4 & 9.6 & 10.1 \\
\hline $\mathrm{X}_{3}(\mathrm{MPa})$ & -0.558 & -1.261 & 0.249 & 0.634 \\
$\mathrm{X}_{2}(\mathrm{MPa})$ & -0.549 & 1.011 & -3.871 & -6.032 \\
$\mathrm{X}_{1}(\mathrm{MPa})$ & 28.85 & 25.41 & 17.52 & 20.91 \\
\hline
\end{tabular}

Table 3 Composite properties and stress-strain input data

\begin{tabular}{|lcccc|}
\hline Sample & GF-PA6,6 & GF-PBT & GF-PPm & GF-PPh \\
\hline Weight fraction $(\%)$ & 32.1 & 29.7 & 30.7 & 30.0 \\
Volume fraction & 0.172 & 0.175 & 0.135 & 0.130 \\
$<\cos ^{2}(\phi)>$ & 0.756 & 0.640 & 0.663 & 0.691 \\
N average length $(\mathrm{mm})$ & 0.331 & 0.498 & 0.731 & 1.101 \\
W average length $(\mathrm{mm})$ & 0.530 & 0.746 & 1.020 & 1.614 \\
Average diameter $(\mu \mathrm{m})$ & 14.0 & 13.8 & 13.4 & 13.4 \\
Strength $(\mathrm{MPa})$ & 162.5 & 138.4 & 95.3 & 66.0 \\
Modulus $(\mathrm{GPa})$ & 9.4 & 10.4 & 7.2 & 7.6 \\
Elongation $(\%)$ & 3.0 & 2.2 & 2.7 & 1.4 \\
& & & & \\
Strain Levels $(\%)$ & $1.0,2.0$ & $0.75,1.5$ & $1.0,2.0$ & $0.5,1.0$ \\
Composite stress 1 $(\mathrm{MPa})$ & 87.9 & 73.4 & 58.1 & 33.4 \\
Composite stress 2 $(\mathrm{MPa})$ & 142.7 & 119.8 & 87.5 & 56.2 \\
Matrix stress 1 $(\mathrm{MPa})$ & 27.9 & 20.0 & 13.9 & 8.7 \\
Matrix stress 2 $(\mathrm{MPa})$ & 51.4 & 37.0 & 21.3 & 15.2 \\
\hline
\end{tabular}

Table 4 Parameters obtained from equation 1 and 2

\begin{tabular}{|lcccc|}
\hline & GF-PA6,6 & GF-PBT & GF-PPm & GF-PPh \\
\hline$\eta_{\text {o }}$ & 0.650 & 0.718 & 0.591 & 0.626 \\
$\tau(\mathrm{MPa})$ & 32.9 & 19.3 & 14.8 & 7.4 \\
$\sigma_{\text {uf }}(\mathrm{GPa})$ & 1.93 & 1.50 & 1.80 & 0.99 \\
\hline
\end{tabular}

Table 5 Input parameters for modelling residual inface radial stress

\begin{tabular}{|lcccc|}
\hline & PA6,6 & PBT & PP & Glass Fibre \\
\hline Coefficient Linear Thermal Expansion $\left(\mu \mathrm{m} / \mathrm{m}^{\circ} \mathrm{C}\right)$ & 110 & 90 & 120 & 5 \\
Modulus $(\mathrm{GPa})$ & 2.9 & 2.8 & 1.5 & 72 \\
Temperature range of stress buildup $\left({ }^{\circ} \mathrm{C}\right)$ & 220 & 160 & 100 & \\
Poisson ratio & 0.35 & 0.35 & 0.35 & 0.22 \\
\hline
\end{tabular}




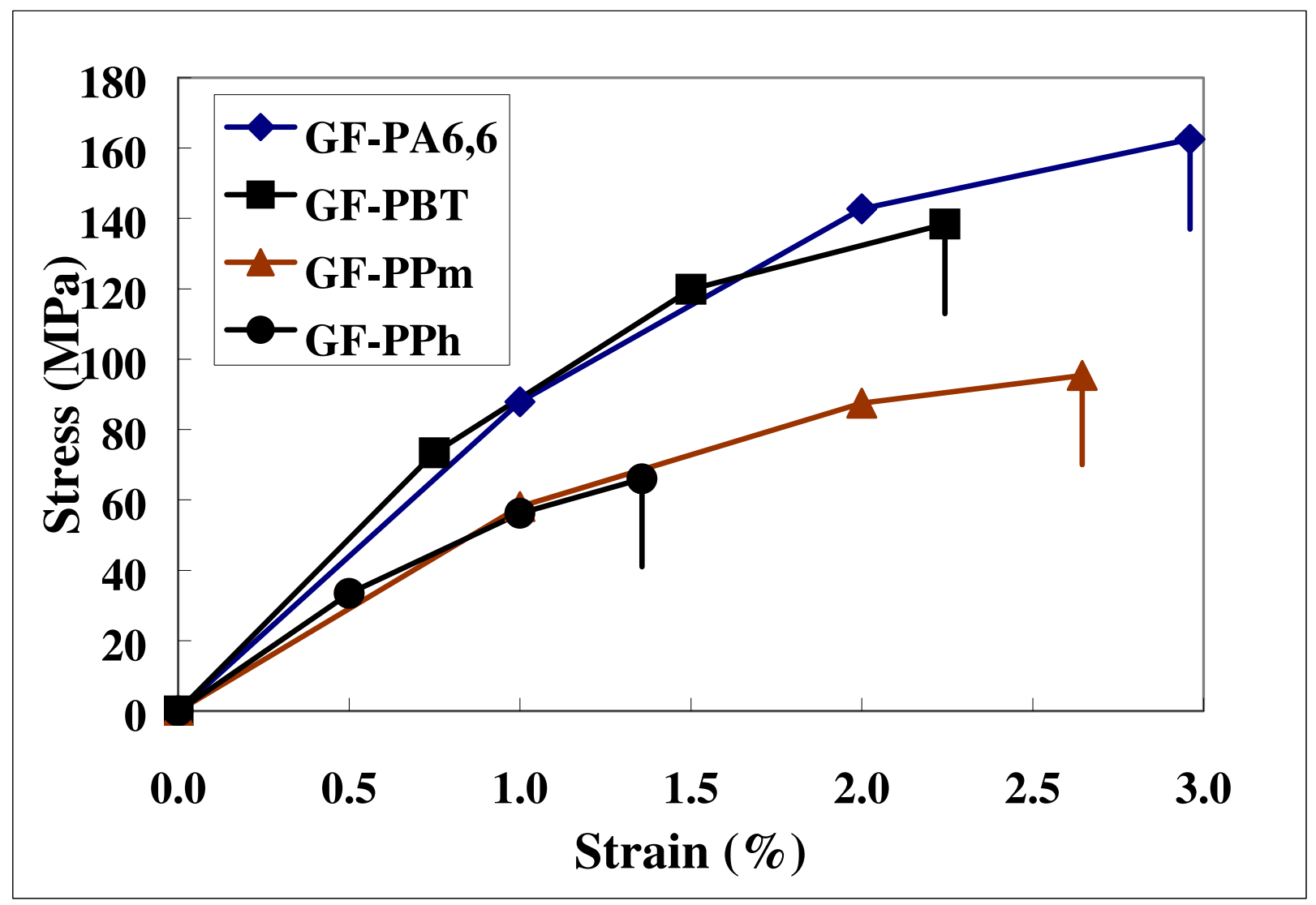

Figure 1 Stress-strain data points from composites

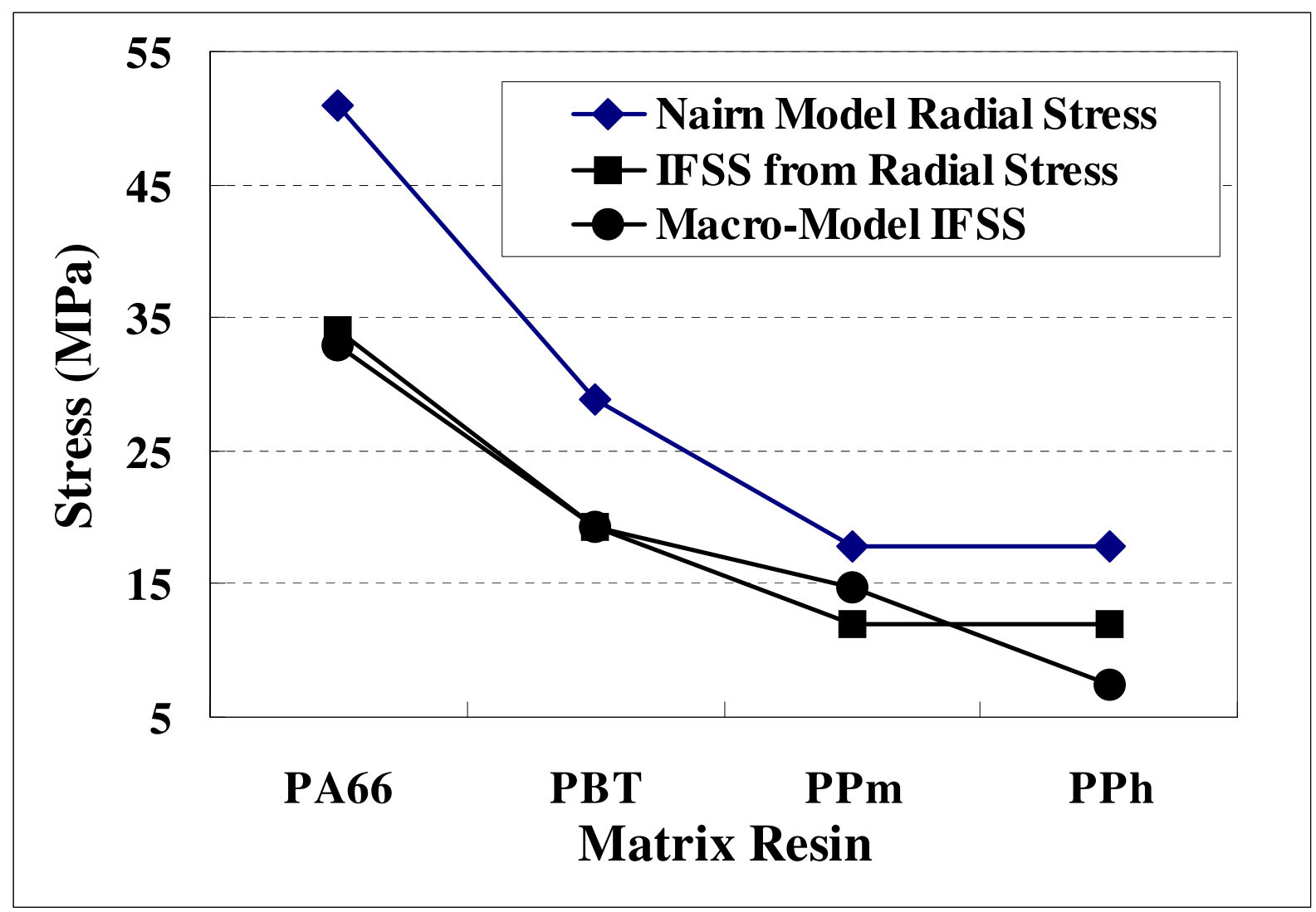

Figure 2 IFSS values from macro-method 


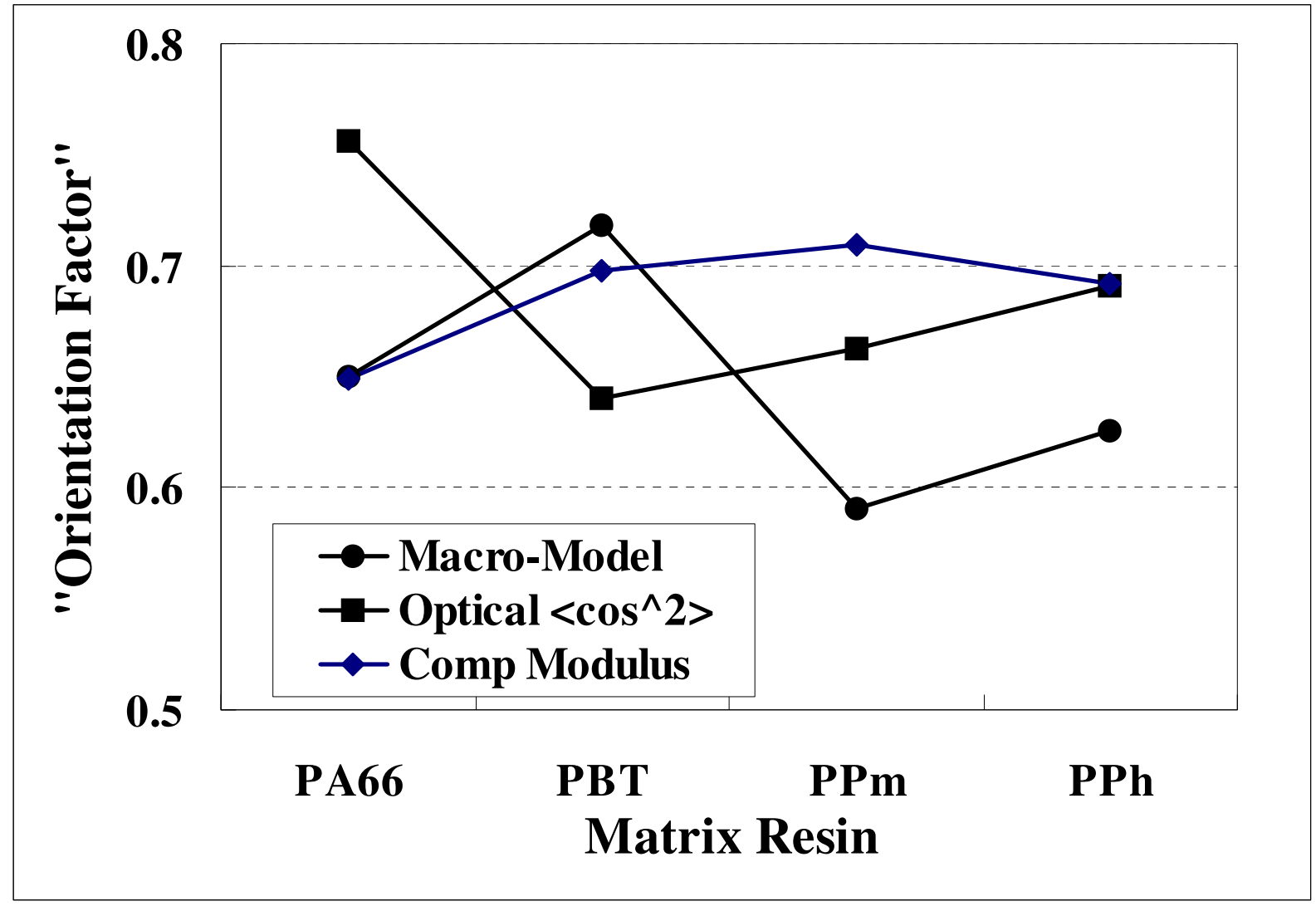

Figure 3 Comparison of orientation parameters

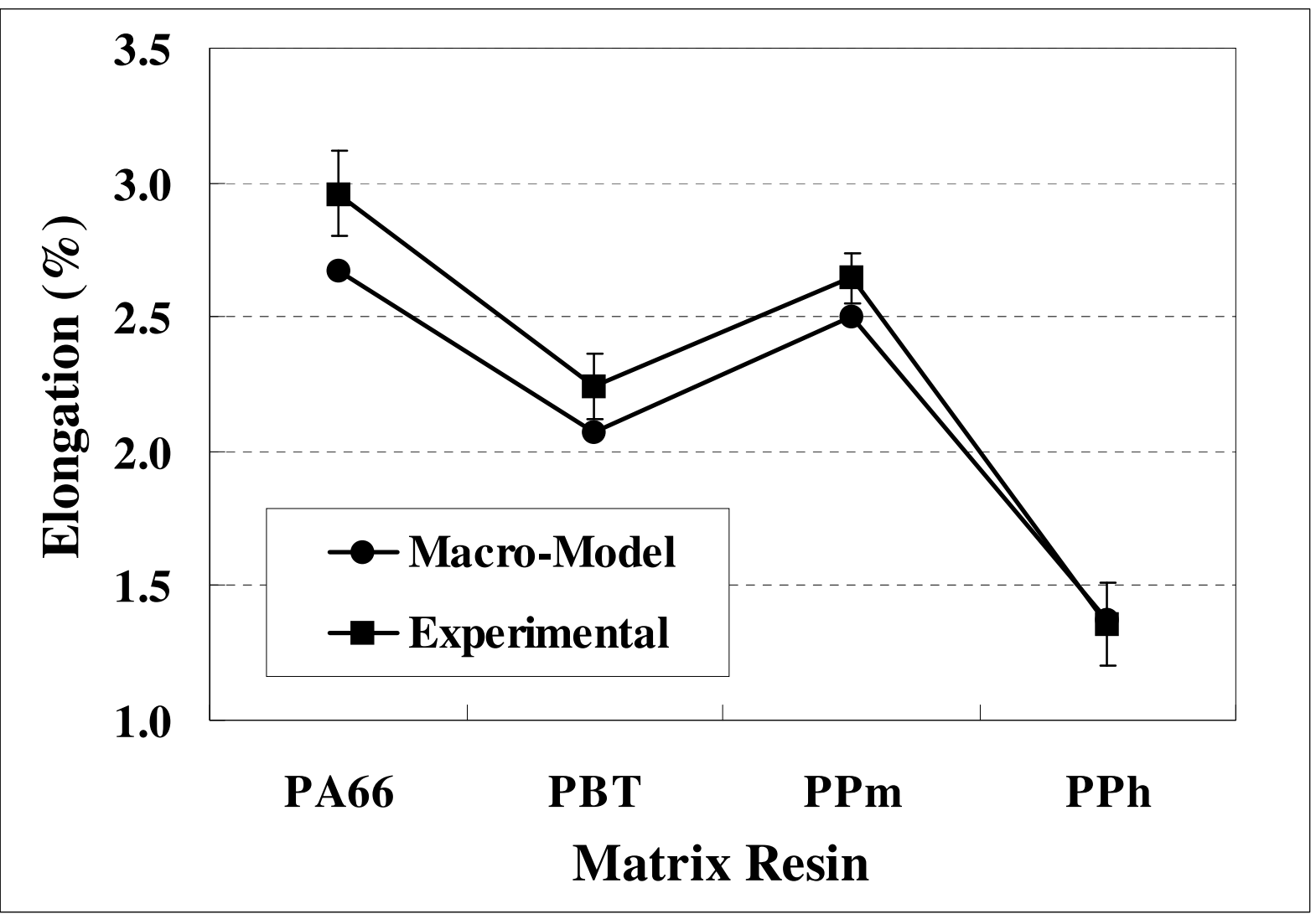

Figure 4 Comparison of macro-method fiber strain and composite failure strain 